The key to the control of blood pressure lies in control of sodium and to a lesser extent watęr balance. Most patients respond to these measures, and at first it was hoped that the blood pressure in all of them could be controlled. ${ }^{4}$ The technique generally employed has been to regulate salt intake at 20-30 mEq per day, to restrict water intake, and to reduce gently the body weight over the first weeks of regular dialysis until some postural hypotension appears. Gradually it has been realized that while most patients respond to this treatment a minority do not. V. Vertes and colleagues, ${ }^{5}$ describing 40 patients on regular dialysis, report that 35 remained with normal blood pressures and could come off all hypotensive therapy, but the other five were radically different. Reduction in body weight combined with rigid sodium restriction failed to reduce the blood pressure to normal. Considerable doses of antihypertensive drugs resulted in brief periods of normal blood pressure during or after dialysis, usually punctuated by hypotension, with a return to high blood pressures after 24 hours. In four of these patients bilateral nephrectomy was performed, with subsequent easy control of blood pressure at normal levels. Other workers ${ }^{6}$ have shown that such patients, once nephrectomy has been performed, respond to sodium depletion exactly as the majority. In Vertes and his colleagues' patients there was no significant difference in any clinical or biochemical feature of the two groups, except for the serum renin concentration. This was raised in the group with resistant hypertension and normal or low in the majority with controllable hypertension.

The clinical observations of most dialysis units agree with those of Vertes and his colleagues, though many would consider that 18 hours per week of dialysis on a twin-coil kidney is insufficient for optimum control of blood pressure. Some would also prefer to believe that the two groups of "saltwater-dependent hypertension" and " renin-dependent hypertension" which Vertes and his colleagues separate sharply may merge into one another.

At a recent meeting of the Dialysis Group and the Renal Association in Glasgow the experiences of several British dialysis and transplantation units in hypertensive control were presented. Most groups found that some of their patients had inadequate control of blood pressure and required moderate doses of hypotensive drugs. This was commonly thought to be due to failure of the patients to stick to rigid salt-andwater restriction. However, a tiny minority of patients had uncontrollable hypertension even when their intake of their salt and water was closely observed. J. J. Brown and his colleagues $^{7}$ presented data from these and other patients on regular dialysis. They showed that plasma renin levels were highest in those with uncontrollable hypertension, reaching on occasion enormous concentrations. As in the patients of Vertes and his colleagues, bilateral nephrectomy brought the blood pressure under easy control. Brown and colleagues also pointed to a problem of particular interest: attempts to

\footnotetext{
1 Bright, R., Guy's Hospital Reports, 1836, 1, 380.

2 Hegstrom, R. M.. Murray, J. S., Pendras, J. P., Burnell, J. M., and Scribner, B. H.. Transactions, American Society for Artificial In-

ternal Organs, 1962, 8, 266.
B Blumberg, A., Hcgstrom, R. M., and Nelp, W. B., in Proceedings of the 1st Conference of the European Dialysis and Transplant Associa tion, 1964, p. 199, ed. D. N. S. Kerr. Amsterdam, Scheltema and

Holima.
- Comty, C., Rottka, H., and Shaldon, S., in Proceedings of the 1st Conference of the European Dialysts and Transplant Association, 1964 p. 209, ed. D. N. S. Kerr. Amsterdam, Scheltema and Holima.

- Vertes, V., Cangiano, J. L., Berman, I. B., and Gould, A., New England fournal of Medicine, 1969, 286, 978.

- Onesti, G., Swartz, C., Ramirez, O., and Brest, A. N., Transactions, American Society for Artificial Internal Organs, 1968, 14, 361.

' Brown, J. J., Curtis, J. R., Lever, A. F., Robertson, J. I. S., de Wardener, H. E., and Wing, A. J., Nephron, 1969, in press.
}

produce more and more salt depletion in this type of patient might lead to higher renin concentrations, with worsening in the level of blood pressure.

Two conclusions emerge from these studies. Firstly, estimation of the concentration of plasma renin is of practical value in patients on regular haemodialysis. Secondly, the volume of the extracellular fluid is directly related to the hypertension in patients with severely impaired or absent renal function. Despite the flood of effective hypotensive drugs now appearing, the simple and old-fashioned control of salt-and-water intake forms a central part of the management of these patients.

\section{Prune-belly Syndrome}

The most obvious anomaly in the condition known as prunebelly is the defect in the abdominal musculature. There is an absence of the lower part of the rectus abdominis and the lower and medial parts of the oblique muscles. It was noted by Fröhlich in 1839. ${ }^{1}$ The appearance of the abdomen, protruding, thin-walled, and with wrinkled skin, gives the syndrome its name.

The muscle defect causes surprisingly little disability. The chief importance of the condition lies in the other features of the syndrome, first described by R. W. Parker in $1895 .^{2}$ The bladder and ureters are usually greatly dilated, there are hydronephrosis and small dysplastic kidneys, and the testes are undescended.

The anomalies of the urinary tract were first thought to be due to urethral obstruction, and indeed urethral atresia may sometimes occur. It was present, together with a patent urachus, in three of a series of 20 cases reported from the Hospital for Sick Children. ${ }^{3}$ But usually no urethral obstruction is found. Further, the enlarged bladder does not show the trabeculation found with urethral obstruction. There is probably a primary defect in the musculature of the bladder and of the ureters. In addition the renal dysplasia is of a type suggesting embryonic dysplasia rather than the effects of back-pressure." No cause of the defects of both abdominal and urinary-tract musculature is apparent. Other anomalies that may be present are talipes equinovarus, congenital heart malformation, and malrotation of the gut.

The aetiology is still obscure. The condition is almost entirely limited to males, and the few girl patients reported do not appear to have exactly the same condition. No family studies have been undertaken, but there is no suggestion that the condition is linked to the $\mathrm{X}$ chromosome, and no reports of two brothers have appeared yet.

The prognosis depends largely on the renal function. Some $20 \%$ of the recorded patients died soon after birth and another $30 \%$ in early childhood. However, a follow-up of 14 patients attending the Mayo Clinic since $1945^{5}$ disclosed five who had passed the age of 20 years. Three of these aged 25, 23, and 21 were still alive, while the other two died at the ages of 28 and 24 . There is no evidence that surgical treatment is helpful, but efforts should be made to prevent renal damage from infection.

\footnotetext{
1 Fröhlich, F. Der Mangel der Muskeln, insbesondere der Seiten bauchmuskeln, 1839. Wurzberg, C. A. Zurn.

2 Parker, R. W., Transactions of the Clinical Society of London, 1895, 28, 201.

3 Williams, D. I., and Burkholder, G. V., fournal of Urology, 1967, 98 , 244.

: Nunn, I. N., and Stephens, F. D., Fournal of Urology, 1961, 86, 782.

5 Burke, E. C., Shin, M. H., and Kelalis, P. P., American fournal of Diseases of Children, 1969, 117, 668
} 